\title{
Um Modelo Matemático via Análise de Regressão para Avaliar a Difusão de Cálcio e de Hidroxila nas Paredes Dentárias
}

G.C. GOMES ${ }^{1}$, J.I.D. PINHEIRO², DME/IM-UFRJ, Cx.P. 68530, 21945-970 Rio de Janeiro, RJ, Brasil

I.C.G. CAMÕES ${ }^{3}$, Faculdade de Odontologia, UFF, RJ, Brasil

M.R. SALLES 4 , Instituto de Química, UFRJ, Rj, Brasil.

\begin{abstract}
Resumo Os objetivos iniciais deste estudo eram descrever e comparar os comportamentos de diferentes grupos de tratamentos odontológicos para difusão de cálcio e hidroxila ao longo do tempo de exposição. Para isso foi importante delinear um experimento que contemplasse adequadamente as diferentes fases (dissolução e difusão) e todas as suas peculiaridades.

Do ponto de vista da modelagem estatística optamos por considerar um modelo único que abrangesse todos os tratamentos simultâneamente, o que foi uma escolha importante para viabilizar a realização dos testes de hipóteses relativos a diferentes modelos encaixantes.

Embora a motivação inicial tenha sido resolver um problema odontológico, este estudo levanta algumas questões metodológicas interessantes que estão discutidas na seção 3.3 .
\end{abstract}

\section{Introdução}

O presente trabalho é uma aplicação estatística na área de Odontologia. O objetivo é analisar como a difusão de Cálcio $\left(\mathrm{Ca}^{2+}\right)$ e o $\mathrm{pH}$ dependem do tempo de exposição para veículos (tratamentos) que viabilizam a passagem do cálcio e da hidroxila através dos canais. O estudo analisa os valores do $\mathrm{pH}$ e do Cálcio, no meio externo às raízes em 10 grupos, onde três são controles e sete são tratamentos usados em endodontia. Para cada um dos 9 grupos, exceto o controle de selamento (com somente um dente), foram observadas 5 réplicas. Os dados foram analisados numa janela terapêutica, nas proximidades da intervenção medicamentosa, à qual

\footnotetext{
${ }^{1}$ gastao@dme.ufrj.br

2 jismael@dme.ufrj.br

${ }^{3}$ belbel@gbl.com.br

${ }^{4}$ milton@iq.ufrj.br
} 
correspondem 414 observações. A técnica utilizada foi a Análise de Regressão sendo as medidas de Cálcio e de $\mathrm{pH}$ as variáveis a explicar. As variáveis explicativas são o tempo e 20 variáveis indicadoras do particular tratamento e da fase: dissolução (antes do tratamento) ou difusão (depois do tratamento). Além dos modelos completos para o cálcio e o $\mathrm{pH}$ tínhamos também interesse em avaliar até que ponto os parâmetros que definem as retas de regressão eram os mesmos para diferentes tratamentos. Consideramos modelos encaixantes para decidir sobre a semelhança dos dois modelos em questão: o completo e o reduzido, o que nos permitiu testar hipóteses de igualdade dos parâmetros (inclinação e/ou intercepto) para diferentes grupos de tratamentos.

\section{Descrição do Experimento}

$\mathrm{O}$ estudo analisa os valores do $\mathrm{pH}$ e do Cálcio, no meio externo às raízes, relativos a 41 dentes pré-molares humanos, cujos canais foram preenchidos por misturas de $\mathrm{Ca}(\mathrm{OH})_{2}$ com diversos veículos, em 23 momentos. Os dentes foram mantidos durante 2855 horas imersos, um a um, em frascos contendo água. As medições foram divididas em duas fases: 1-Dissolução - todos os condutos foram mantidos vazios e abertos durante 1168 horas; 2-Difusão - os espécimes foram divididos em 10 grupos, dos quais 3 são grupos de controle: Grupo1 - água, Grupo2 - selamento, Grupo3 - difusão do conduto aberto e 7 são grupos experimentais, compostos por 5 dentes cada, cujos condutos foram preenchidos por pasta de $\mathrm{Ca}(\mathrm{OH})_{2}$ associado a um particular veículo usado em endodontia. Mais detalhes sobre o experimento estão nas referências [2] e [3].

\subsection{Fases da Medição}

As medições foram basicamente divididas em duas fases: "Dissolução - $1^{a}$ fase" Os canais foram mantidos vazios e sem selamento cervical por um período de 1168 horas (48 dias e 16 horas) para estabilizar as perdas de $\mathrm{Ca}^{2+}$ e de $\mathrm{OH}-$ da estrutura dentária para o meio aquoso. "Difusão $-2^{a}$ fase" - Nesta fase, foi realizado o preenchimento dos condutos com pasta de $\mathrm{Ca}(\mathrm{OH})_{2}$ associado a 7 veículos.

\subsection{Os Grupos}

Os 41 dentes estudados foram alocados em 10 grupos:

- Os 3 Grupos Controle: Grupo 1 - Controle da água - mantidos sem dentes durante todo o experimento, com o intuito de monitorar a entrada de $\mathrm{CO}_{2}$ no meio; Grupo 2 - Controle do selamento - o dente teve a superfície externa totalmente coberta por Araldite ultra-rápido para ratificar a eficiência do selamento; Grupo 3 - Controle da dissolução - os dentes foram mantidos vazios (um em cada frasco) durante todo o experimento, com a finalidade de permitir o acompanhamento de sua dissolução. 
- Os 7 Grupos Experimentais - os grupos foram compostos por 5 dentes, sendo colocado 1 dente em cada frasco, cujos condutos foram preenchidos com pasta, resultante da mistura de $\mathrm{Ca}(\mathrm{OH})_{2}$ e 7 veículos.

Quando os preenchimentos foram concluídos, os selamentos cervicais foram colocados, exceto em um - "controle de selamento", que teve sua superfície completamente coberta por Araldite para checar a eficácia do selamento contra a difusão de $\mathrm{Ca}^{2+}$ e de OH-.

Mais especificacmente, os grupos considerados foram os seguintes:

- Grupo 1: Controle de água (frascos: 42, 43, 44, 45,46)

- Grupo 2: Controle de selamento (frasco: 1)

- Grupo 3: Controle de dente - Apenas dissolução (frascos: 5, 6, 7, 8, 9)

- Grupo 4: Soro fisiológico $+\mathrm{Ca}(\mathrm{OH})_{2}($ frascos: $17,18,19,20,21)$

- Grupo 5: Calen (frascos: 4, 37, 38, 39, 40)

- Grupo 6: Glicerina + PMCC + $\mathrm{Ca}(\mathrm{OH})_{2}($ frascos: 2, 33, 34, 35, 36)

- Grupo 7: PMCC + $\mathrm{Ca}(\mathrm{OH})_{2}$ (frascos: $\left.3,29,30,31,32\right)$

- Grupo 8: Glicerina + $\mathrm{Ca}(\mathrm{OH})_{2}$ (frascos: $\left.15,16,22,23,24\right)$

- Grupo 9: Glicerina + TCF + $\mathrm{Ca}(\mathrm{OH})_{2}$ (frascos: 25, 26, 27, 28, 41)

- Grupo 10: Anestésico $+\mathrm{Ca}(\mathrm{OH})_{2}$ (frascos: 10, 11, 12, 13, 14)

Queremos analisar como a difusão de Cálcio (dc) e o $\mathrm{pH}$ dependem do tempo para cada um dos grupos acima. Mais detalhes sobre a formação dos grupos estão em Camões [3].

\subsection{Os Tempos de Medição}

Para cada solução aquosa em diferentes intervalos de tempo foram feitas medições dos valores de:

- pH, por meio de um eletrodo combinado acoplado a um pH-metro,

- $\mathrm{Ca}^{2+}$, a concentração de cálcio, por meio de um espectrômetro de absorção atômica.

Para cada um dos 46 frascos foram medidos a difusão de cálcio e o $\mathrm{pH}$ em 23 momentos contados (em horas) a partir do início do experimento: 0, 43, 91, 165, $230,406,552,671,839,1054,1168,1242,1291,1361,1433,1513,1601,1680,1849$, 2015, 2184, 2520, 2855. O tratamento foi efetivamente iniciado no instante 1168. Até este momento o experimento tinha o objetivo de controlar os dentes e o meio aquoso. Para cada um dos 10 grupos foram analisadas 5 réplicas, ou seja, dentes, um em cada frasco diferente, os quais foram examinados ao longo do tempo. Para o Grupo 2, excepcionalmente, foi analisado um só dente. Com exceção do Grupo 1, controle de água, as réplicas são dentes imersos no meio aquoso. 


\section{Análise Estatística}

A técnica estatística usada foi a Análise de Regressão sendo a difusão de Cálcio e o pH as variáveis a explicar. As variáveis explicativas são o tempo (t) e algumas variáveis indicadoras, ou seja, variáveis que assumem o valor "um" quando a característica está presente e o valor "zero" quando ela está ausente. Um bom exemplo do uso de variáveis indicadoras pode ser visto em Weisberg [6](pag.177-183). As 20 variáveis indicadoras aqui usadas são:

- $\mathrm{A}_{i}$ para indicar se a observação pertence ao Grupo i, $(\mathrm{i}=1, \ldots, 10)$, antes do tratamento;

- $\mathrm{D}_{i}$ para indicar se a observação pertence ao Grupo i, $(\mathrm{i}=1, \ldots, 10)$, depois do tratamento;

As variáveis indicadoras $A_{1}, \cdots, A_{10}, D_{1}, \cdots, D_{10}$ são excludentes entre si: quando para uma observação uma delas assume o valor um, as outras assumem o valor zero.

Para efeito terapêutico foi de interesse analisar os dados numa "janela" em torno do momento da aplicação do tratamento que corresponde a medidas nos tempos: 671, 839, 1054, 1168 antes do tratamento e nos tempos: 1242, 1291, 1361, 1433 e 1513 depois do tratamento. Assim nossa massa de dados foi restrita a esta janela terapêutica à qual correspondem 414 (9 tempos vezes 46 frascos) observações sendo dc e $\mathrm{pH}$ as variáveis dependentes e sendo as 21 variáveis explicativas: t (tempo), $A_{1}, \cdots, A_{10}, D_{1}, \cdots, D_{10}$.

\subsection{Análise Exploratória}

A seguir apresentamos, para efeito descritivo das observações, os gráficos de cada grupo de tratamento para o Cálcio e para o pH. Foram tomados alguns cuidados para facilitar a comparação entre os grupos em cada caso:

- o eixo vertical onde é medida a variável resposta foi padronizado na mesma escala(intervalo de valores) para todos os grupos de tratamento: 0.0 e 3.0 para o cálcio e 5.0 e 8.0 para o $\mathrm{pH}$;

- em cada gráfico há uma marca vertical que especifica o momento da intervenção: antes dessa marca temos a dissolução e depois da marca a difusão. 
3.1.1. Gráficos do Tempo versus Cálcio
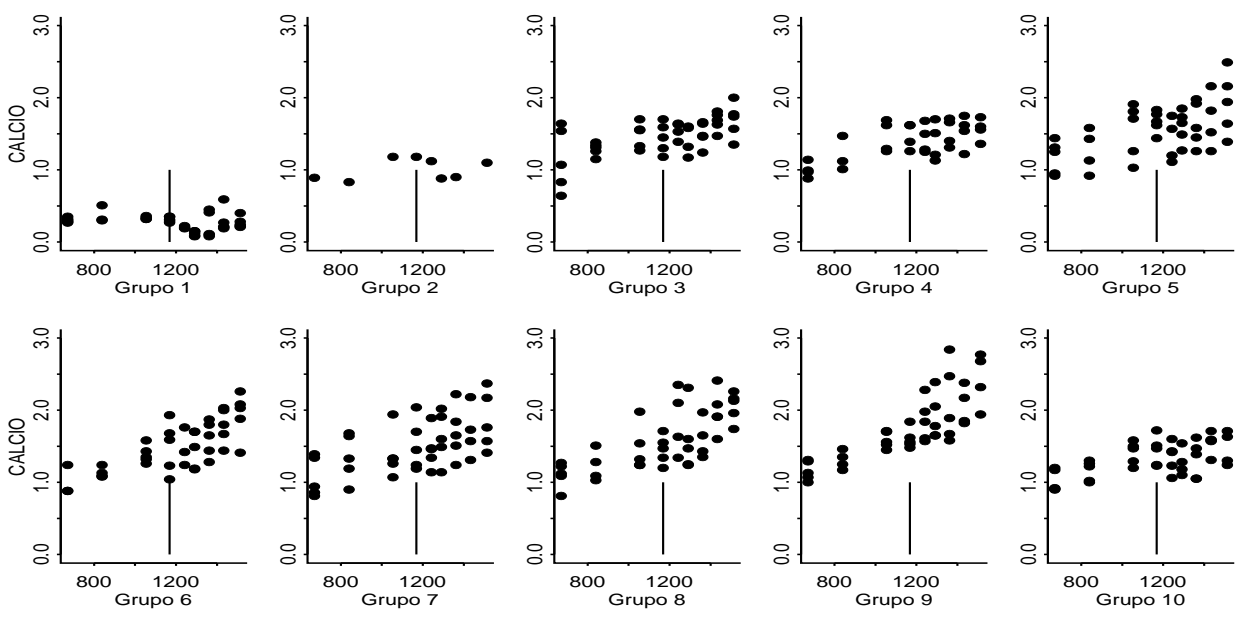

3.1.2. Gráficos do Tempo versus $\mathrm{pH}$
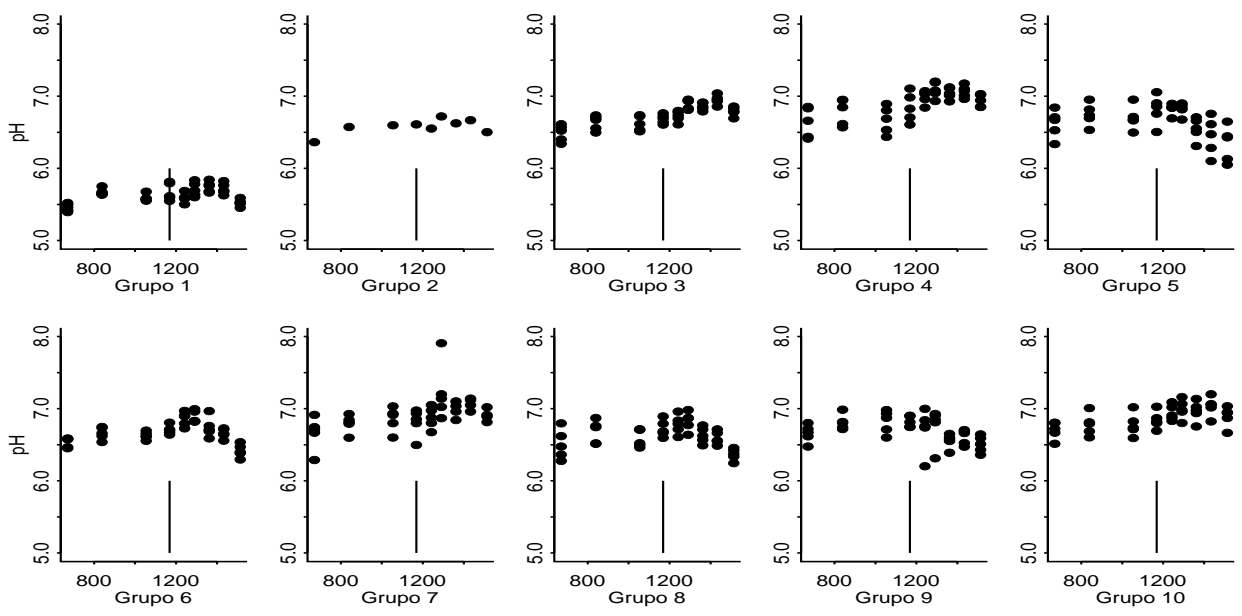

Começaremos a nossa análise dos dados com os modelos completos, equação de regressão (3.1) para:

- o Problema I: y=dc, difusão de cálcio e

- o Problema II: $\mathrm{y}=\mathrm{pH}$, difusão de hidroxila.

$$
\begin{aligned}
& y=\alpha_{1} A_{1}+\cdots+\alpha_{10} A_{10}+\beta_{1} A_{1} t+\cdots+\beta_{10} A_{10} t \\
& +\alpha_{1} D_{1}+\cdots+\alpha_{10} D_{10}+\beta_{1} D_{1} t+\cdots+\beta_{10} D_{10} t \text {. }
\end{aligned}
$$


Basicamente estes modelos ajustam 10 retas de regressão antes da aplicação do tratamento, uma para cada Grupo $i=1,2, \ldots, 10$ e , outras 10 retas, uma para cada grupo, depois do tratamento. Com estas 20 retas estamos descrevendo o comportamento de cada grupo ao longo do tempo, antes e depois da aplicação do tratamento. Os parâmetros da equação (3.1) foram estimados. Quando fizemos uma análise de diagnóstico dos resíduos de cada caso, nesta primeira tentativa, observamos que havia 4 pontos (entre os 414) com comportamento muito discrepante. Essa análise envolve as estatísticas de Cook, DFFITS, $\mathrm{h}_{i i}$, resíduos studentizados, etc.. Maiores detalhes podem ser encontrados nas referências [1] e [4].

Os 4 pontos discrepantes são os mesmos para o cálcio e para o $\mathrm{pH}$, sendo que esta discrepância é bem mais visível no caso do $\mathrm{pH}$, como pode ser visto nos gráficos de resíduos a seguir.

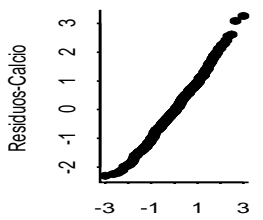

Quantis da NORMAL
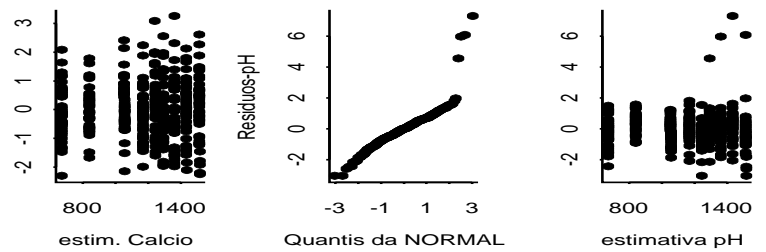

Observamos que estes pontos eram todos do frasco 29, do Grupo 7. Na investigação sobre o que tinha ocorrido, nos foi informado que para este dente houve problemas com o selamento. Decidimos eliminar da análise as medidas deste dente e a nova massa de dados passou então de 414 para 405 observações.

A seguir apresentaremos as estimativas dos parâmetros $\alpha$ e $\beta$ das equações (3.1) usando a massa de dados revisada. Ao fim de cada modelo exibiremos medidas sobre a qualidade do ajuste. Maiores esclarecimentos estatísticos sobre estas medidas podem ser vistos em Montgomery \& Peck [5].

Tabela 1: Problema I: Estimação dos $\alpha$ e $\beta$ da equação (3.1) com y=dc

\begin{tabular}{c||c|c||c|c||c|c||c|c||}
\multicolumn{1}{c||}{} & \multicolumn{4}{c||}{ Antes do Tratamento } & \multicolumn{3}{c||}{ Depois do Tratamento } \\
\cline { 2 - 9 } Grupo & \multicolumn{2}{c|}{$\alpha$} & \multicolumn{2}{c||}{$\beta$} & \multicolumn{2}{|c||}{$\alpha$} & \multicolumn{2}{|c}{$\beta$} \\
\cline { 2 - 9 } & Coef. & p-val. & Coef. & p-val. & Coef. & p-val. & Coef. & p-val. \\
\hline 1 & 0.319 & 0.256 & 0.0000 & 0.978 & -0.491 & 0.518 & 0.0005 & 0.345 \\
2 & 0.331 & 0.589 & 0.0007 & 0.250 & 0.699 & 0.668 & 0.0002 & 0.853 \\
3 & 0.720 & 0.009 & 0.0007 & 0.021 & 0.413 & 0.576 & 0.0008 & 0.117 \\
4 & 0.331 & 0.286 & 0.0010 & 0.002 & 0.649 & 0.404 & 0.0006 & 0.281 \\
5 & 0.460 & 0.097 & 0.0010 & 0.000 & -0.575 & 0.430 & 0.0016 & 0.002 \\
6 & 0.293 & 0.363 & 0.0010 & 0.002 & -1.007 & 0.185 & 0.0019 & 0.001 \\
7 & 0.717 & 0.020 & 0.0007 & 0.037 & 0.619 & 0.426 & 0.0007 & 0.188 \\
8 & 0.587 & 0.034 & 0.0008 & 0.007 & -0.081 & 0.911 & 0.0014 & 0.009 \\
9 & 0.514 & 0.064 & 0.0010 & 0.001 & -0.542 & 0.461 & 0.0019 & 0.001 \\
10 & 0.425 & 0.120 & 0.0009 & 0.002 & 0.321 & 0.664 & 0.0008 & 0.145 \\
\hline
\end{tabular}

$\mathrm{N}=371$, com 34 observações perdidas; $\mathrm{F}=321.3$ com 40 e 331 gl, $\mathrm{p}$-valor $=0,000$;

$\mathrm{R}^{2}=0.9749 ; \mathrm{SQR}=20.016$ 
Tabela 2: Problema II: Estimação dos $\alpha$ e $\beta$ da equação (3.1) com $\mathrm{y}=\mathrm{pH}$

\begin{tabular}{c||c|c||c|c||c|c||c|c||}
\multicolumn{1}{c||}{} & \multicolumn{4}{c||}{ Antes do Tratamento } & \multicolumn{3}{c||}{ Depois do Tratamento } \\
\cline { 2 - 9 } \multicolumn{1}{c|}{ Grupo } & \multicolumn{2}{c||}{$\alpha$} & \multicolumn{2}{c||}{$\beta$} & \multicolumn{2}{c||}{$\alpha$} & \multicolumn{2}{c||}{$\beta$} \\
\cline { 2 - 9 } & Coef. & p-val. & Coef. & p-val. & Coef. & p-val. & Coef. & p-val. \\
\hline 1 & 5.30 & 0.00 & 0.0003 & 0.05 & 6.01 & 0.00 & 0.0003 & 0.37 \\
2 & 6.12 & 0.00 & 0.0005 & 0.21 & 7.00 & 0.00 & 0.0003 & 0.65 \\
3 & 6.30 & 0.00 & 0.0003 & 0.04 & 6.53 & 0.00 & 0.0002 & 0.41 \\
4 & 6.49 & 0.00 & 0.0003 & 0.10 & 7.33 & 0.00 & 0.0002 & 0.43 \\
5 & 6.44 & 0.00 & 0.0003 & 0.06 & 9.30 & 0.00 & -0.0020 & 0.00 \\
6 & 6.38 & 0.00 & 0.0003 & 0.09 & 8.99 & 0.00 & -0.0017 & 0.00 \\
7 & 6.41 & 0.00 & 0.0004 & 0.04 & 6.74 & 0.00 & 0.0002 & 0.59 \\
8 & 6.34 & 0.00 & 0.0003 & 0.06 & 8.81 & 0.00 & -0.0016 & 0.00 \\
9 & 6.50 & 0.00 & 0.0003 & 0.06 & 7.74 & 0.00 & -0.0008 & 0.00 \\
10 & 6.56 & 0.00 & 0.0002 & 0.14 & 7.13 & 0.00 & 0.0001 & 0.67 \\
\hline
\end{tabular}

$\mathrm{N}=405 ; \mathrm{F}=321.3$ com 40 e 365 gl, p-valor $=0,000 ; \mathrm{R}^{2}=0.9996 ; \mathrm{SQR}=6.795$.

\subsection{Análise Estatística Visando a Identificação de Grupos com Comportamento Equivalente}

Além desta formulação geral tínhamos também como interesse juntar grupos que apresentassem comportamentos equivalentes conforme sugerido pela análise exploratória precedente, aliada a considerações sobre a própria natureza físico-quimica dos tratamentos em exame. Para isso consideramos modelos aninhados (Weisberg [6]). Para decidirmos sobre a semelhança dos dois modelos em questão, o completo e o reduzido (com menos variáveis explicativas), foi testada uma hipótese de igualdade dos parâmetros (inclinação e/ou intercepto) para diferentes tratamentos. A estatística de teste $\mathrm{F}$ se baseou nas SQR (soma dos quadrados dos resíduos) dos modelos em questão com os seus respectivos gl (graus de liberdade):

$$
F_{C R}=\frac{\left(S Q R_{R}-S Q R_{C}\right) g l_{C}}{S Q R_{C}\left(g l_{R}-g l_{C}\right)},
$$

onde, o índice $\mathrm{C}$ significa modelo completo e o $\mathrm{R}$ significa reduzido.

O objetivo é juntar grupos sem que aumente significativamente a SQR, sendo a medida para esta verificação a estatística expressa pela equação (3.2).

Procuramos identificar grupos para os quais houvesse uma grande proximidade $\operatorname{dos} \beta$ (inclinação) e, evidentemente, para os quais os tratamentos apresentassem afinidade do ponto de vista terapêutico. Testamos então a hipótese de que os $\beta$ correspondentes a esses grupos são iguais. Nos casos em que a hipótese nula (de igualdade $\operatorname{dos} \beta$ ) foi aceita, podemos considerar que, após a aplicação do tratamento, os comportamentos dos diversos grupos envolvidos com relação ao tempo são estatisticamente equivalentes. $\mathrm{O}$ p-valor correspondente à estatística acima mencionada é o instrumento que nos permitiu decidir pela aceitação ou pela rejeição dessa hipótese. 
O mesmo tipo de raciocínio também foi tentado com relação aos $\alpha$ (intercepto), porém, como estes são muito sensíveis a pequenas variações dos $\beta$, neste caso não obtivemos sucesso em termos da redução dos modelos.

Com base nas afinidades que decorrem de considerações físico-quimícas foram testados diferentes hipóteses de igualdade dos coeficientes do modelo completo. Apresentamos a seguir, para cada um dos dois problemas, os modelos que se revelaram mais satisfatórios. Em ambas as equações abaixo, tanto para a difusão de cálcio como para o $\mathrm{pH}$, o símbolo $\mathrm{A}_{3 a 10}$ coresponde a uma única função indicadora (antes do tratamento) para os grupos de 3 a 10. Analogamente, o símbolo $\mathrm{D}_{5689}$ coresponde a uma única função indicadora (depois do tratamento) para os grupos $5,6,8$ e 9 .

\subsubsection{Um Modelo Mais Adequado para o Problema I}

$$
\begin{aligned}
d c= & 0.326 A_{1}+1.020 A_{2}+0.521 A_{3}+0.465 A_{4}+0.597 A_{5} \\
& +0.441 A_{6}+0.524 A_{7}+0.494 A_{8}+0.598 A_{9}+0.437 A_{10} \\
& +0.0009 A_{3 a 10} t \\
& +0.225 D_{1}+1.000 D_{2}+1.570 D_{3}+1.486 D_{4}-0.681 D_{5} \\
& -0.690 D_{6}+1.642 D_{7}-0.528 D_{8}-0.270 D_{9}+1.394 D_{10} \\
& +0.0017 D_{5689} t
\end{aligned}
$$

$\mathrm{SQR}=20.763 ;$ com $\mathrm{F}_{C R}=0.686$ com 18 e 331 gl, p-valor $=0.8251$.

Os resultados da equação (3.3) nos levam a concluir que para o Problema I (difusão do cálcio) o modelo que iguala os $\beta$ 's antes e depois do tratamento da maneira indicada acima é mais adequado do que o modelo completo, já que ele envolve um número bem menor de parâmetros a serem estimados sem aumentar a SQR.

\subsubsection{Um Modelo Mais Adequado para o Problema II}

$$
\begin{aligned}
p H= & 5.599 A_{1}+6.536 A_{2}+6.333 A_{3}+6.461 A_{4}+6.446 A_{5} \\
& +6.356 A_{6}+6.468 A_{7}+6.348 A_{8}+6.503 A_{9}+6.500 A_{10} \\
& +0.0003 A_{3 a 10} t \\
& +5.656 D_{1}+6.613 D_{2}+6.843 D_{3}+7.025 D_{4}+8.661 D_{5} \\
& +8.777 D_{6}+6.970 D_{7}+8.705 D_{8}+8.692 D_{9}+6.965 D_{10} \\
& -0.0015 D_{5689} t
\end{aligned}
$$

$\mathrm{SQR}=7.129 ;$ com $\mathrm{F}_{C R}=1.020$ com 18 e 365 gl, p-valor $=0.4359$

Como no Problema I, a equação (3.4), que além de igualar os $\beta$ antes do tratamento também iguala os $\beta$ depois do tratamento para os grupos do Calen e os que contém glicerina como componente, mostrou-se mais adequada para o Problema II. 


\subsection{Discussão da Metodologia Utilizada}

\subsubsection{Modelo Único Versus 20 Modelos Separados}

Uma alternativa à abordagem aqui utilizada teria sido ajustar 20 modelos diferentes, um para cada grupo de tratamento e para cada fase. Caso isso tivesse sido feito, os valores estimados dos coeficientes de cada reta teriam sido exatamente os mesmos aqui obtidos. Qual seria então a diferença entre essas duas possíveis abordagens?

A abordagem obtida neste trabalho pressupõe que a variabilidade dos pontos em torno de cada uma das retas de regressão é sempre a mesma. Evidentemente não haveria nenhuma razão para que isso acontecesse caso tivéssemos optado pelo outro caminho.

Optamos por construir um modelo único devido à nossa intenção de testar a igualdade dos coeficientes (linear e angular) para diferentes grupos, o que era um objetivo importante do trabalho. A razão que nos fez optar por um modelo mais simples, onde a variância do erro é única para todos os tratamentos, foi economizar graus de liberdade na estimação dos parâmetros.

\subsubsection{A Premissa de Normalidade do Erro}

A validade dos testes de hipótese realizados neste trabalho depende da suposição de que o erro segue uma distribuição normal. Dado que esses testes eram o núcleo principal de interesse da pesquisa, foi importante verificar a validade dessa premissa com base na análise dos resíduos. Os plots de resíduos contra os quantis da normal se revelaram bastantes satisfatórios, principalmente depois que foram descartadas as observações correspondentes ao dente 29 para o qual se verificou que houve problemas no selamento.

\subsubsection{Questionando a Independência Entre as Observações}

Dado que no nosso caso um mesmo dente foi observado em vários momentos ao longo do tempo era natural que surgisse uma certa preocupação com a possibilidade de ocorrência de autocorrelação no erro. Entretanto esse questionamento fica amenizado na medida em que foram consideradas réplicas ( 5 dentes para cada tratamento), o que deve ter concorrido para minimizar esse possível desvio em relação às premissas da modelagem.

\subsubsection{Importância da Análise dos Resíduos}

Vale a pena ressaltar que neste estudo, se não tivesse sido feita uma análise criteriosa dos resíduos, não teria sido detectado o comportamento atípico de algumas observações e consequentemente, as conclusões relativas ao Grupo 7 e suas relações com os demais teriam sido outras. Felizmente com base nas suspeitas levantadas pela análise dos resíduos foi possível fazer com que o experimentador pudesse resgatar a ocorrência de problemas durante o experimento com um dos dentes, cujas medições foram eliminadas do conjunto de dados. 


\title{
4. Conclusão
}

Os nossos resultados mostram que a inclinação das retas de regressão que expressa a dependência do pH com relação ao tempo antes da intervenção terapêutica é a mesma para todos os tratamentos, como era de se esperar.

O mesmo ocorreu para a difusão de cálcio, a menos do fato de que para o Grupo 1 (controle de água), para o qual não há a presença de dente e para o Grupo 2 (controle de selamento), não foi possível aceitar a hipótese de igualdade entre a inclinação antes a ele correspondente e as inclinações antes correspondentes aos demais tratamentos.

Além disso, concluímos também que as inclinações das retas de regressão que expressam a dependência entre o pH e o tempo após a intervenção terapêutica são estatisticamente equivalentes entre si para os três grupos com presença de glicerina e do Calen. A mesma conclusão se aplica no caso de difusão de cálcio.

\begin{abstract}
Our initial goals were to describe and compare the behavior of different treatment groups for calcium diffusion and $\mathrm{pH}$, along exposure time. The design of an adequate experiment was important in order to enable us to analyze all the peculiarities of the phenomenon including its two phases, namely, dissolution and diffusion. From the statistical point of view, we decided to build a unique model putting together simultaneously all the treatment groups. That was an important decision since it made possible for us to test hypotheses on different nested models. Although our initial motivation was to approach an odontological problem, this study has raised some interesting methodological issues which we discuss in Section 3.3 .
\end{abstract}

\section{Referências}

[1] D.A. Belsley, E. Kuh e R.E. Welsch, "Regression Diagnostics", Wiley, New York, 1980.

[2] I.C.G. Camões, O. Chevitarese, N.S. Almeida, M.R. Salles e G.C. Gomes, "Diffusion of Calcium through Dentin", Journal of Endodontics, 22, No. 11 (1996), 590-595.

[3] I.C.G.Camões, "Associações Químicas com $\mathrm{Ca}(\mathrm{OH})_{2}$ - Estudo da Difusão de $\mathrm{Ca}^{2+}$ e $\mathrm{OH}^{-}$Através da Dentina", Tese de Doutorado, Faculdade de Odontologia, UFRJ, Rio de Janeiro, RJ, 2000.

[4] R.D. Cook and S. Weisberg, "Residuals and Influence in Regression", Chapman and Hall, New York, 1982.

[5] D.C. Montgomery and E.A. Peck, "Introduction to Linear Regression Analysis", John Wiley and Sons, 1982.

[6] S. Weisberg, "Applied Linear Regression", second edition, John Wiley and Sons, 1985. 